\title{
High-resolution photoemission studies of quasi-one-dimensional systems
}

\author{
D. MALTERRE, B. DARDEL, M. GRIONI, P. WEIBEL and Y. BAER \\ Institut de Physique de Neuchâtel, 1 rue A.-L. Breguet, Neuchâtel, Switzerland
}

\begin{abstract}
High resolution photoemission spectroscopy is exploited for the study of phase transitions in low-dimensional systems. We show that 1D-metals do not exhibit a finite spectral density at the Fermi level. Different mechanisms are invoked to explain this striking behaviour.
\end{abstract}

\section{Introduction}

Photoemission spectroscopy gives information on the electronic structure on a large energy scale (a few eV). However, the recent development of high-resolution photoemission allows a direct determination of the low-energy part of the excitation spectrum which is directly responsible for most physical properties of solids. In this paper, we will illustrate the potentiality of photoemission spectroscopy for the study of low-energy excitations in quasi-one-dimensional systems.

In low-dimension $(D<3)$, electronic transitions, like the charge-density-wave transition (CDW), are often observed. In particular, the one-dimensionality is characterized by several features which qualitatively differ from those at higher dimensionalities. As demonstrated by Peierls [1], the metallic state is unstable with respect to a lattice distortion yielding the formation of an insulating ground state. Nevertheless, with short range interactions, a I-D system cannot present a thermodynamic transition at finite temperature, owing to the very large fluctuations associated with the 1-D character [2]. The electronic correlations are also known to be very unusual [3]; the simple model of Fermi liquid which generally accounts for the excitations in 2- and 3-D materials is no longer valid in 1-D systems where an infrared catastrophe leads to the Luttinger liquid pathology [4]. In real 1-D materials, electronic transitions are actually observed suggesting that transverse interactions play a significant role. However, in systems where these interactions are weak, the main characteristic features of one-dimensionality remain. 


\section{Peierls transition in $1 D$ materials}

Metallic quasi-one-dimensional materials usually exhibit an electronic transition toward a semiconducting phase at low temperature. This transition, called the Peierls transition, is accompanied by the appearance of charge-density-waves and a distortion of the lattice and results from the instability of the electron gas at a wave vector $q=\mathbf{2} \mathbf{k}_{F}$ ( $\mathbf{k}_{\mathrm{F}}$ is the Fermi wave vector). A mean field treatment predicts a modification of the spectral density similar to that encountered in the BCS theory of superconductors [5]. In going from the metallic to the semiconducting phases, a transfer of spectral weight from the vicinity of $E_{F}$ to lower energy is expected, reflecting the opening of the Peierls gap.

The molybdenum blue bronze $\left(\mathrm{K}_{0.3} \mathrm{MoO}_{3}\right)$ belongs to this class of materials. It is a metallic oxide with a crystalline structure with chains of $\mathrm{MoO}_{6}$ clusters forming slabs separated by alkali atoms. A metal-insulator transition is exhibited at $180 \mathrm{~K}$ as shown in many experimental studies [6-8] with a Peierls gap estimated between 100-150 meV from optical measurements [8].

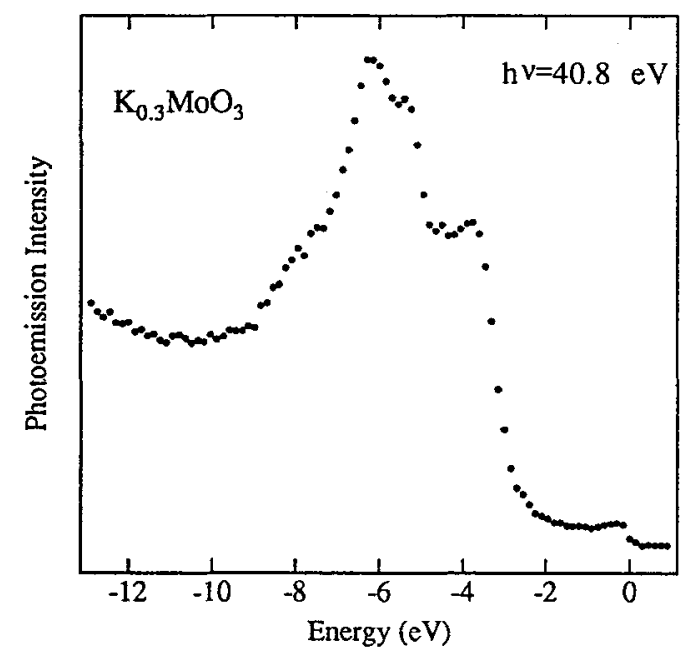

rigure 1: rnotoemission spectrum

of $\mathrm{K}_{0.3} \mathrm{MoO}_{3}$ (hv $=40.8 \mathrm{eV}$ )

Figure 2: Photoemission spectra

of $\mathrm{K}_{0.3} \mathrm{MoO}_{3}$ and $\left(\mathrm{TaSe}_{4}\right)_{2} \mathrm{I}$.

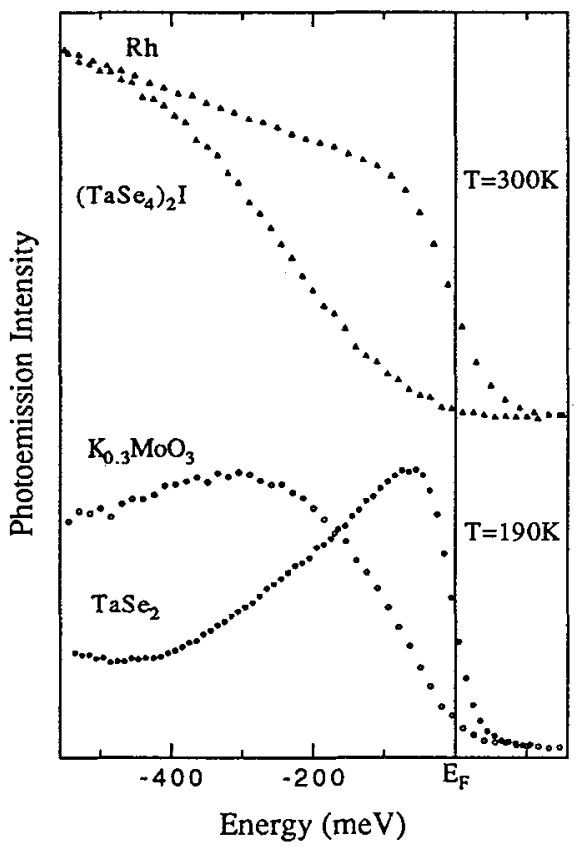

\subsection{The metallic phase}

First let us consider the metallic phase above the Peierls temperature. As shown in figure 1, the UV-photoemission spectrum of this compounds is dominated by the valence band structure between -2 and $-10 \mathrm{eV}$. An additional small feature appears near the Fermi level and reflects the conduction band which results from the formation of hybridized Mo $4 \mathrm{~d}$ and $\mathrm{O} 2 \mathrm{p}$ states [9]. This band, empty in the $\mathrm{MoO}_{3}$ oxide, is partially filled by the alkali s electrons in $\mathrm{K}_{0.3} \mathrm{MoO}_{3}$. In figure 2, we present photoemission spectra in the vicinity of the Fermi level. Surprisingly, the spectrum of $\mathrm{K}_{0.3} \mathrm{MoO}_{3}$ in its metallic phase does not exhibit 
the step at the Fermi energy which is always characteristic of a metal [10]. A similar result is obtained in the CDW compound $\left(\mathrm{TaSe}_{4}\right)_{2} \mathrm{I}\left(\mathrm{T}_{\mathrm{P}}=263 \mathrm{~K}\right)$. This behaviour strongly contrasts with the situation observed for higher dimensionalities as illustrated by the spectra of $\mathrm{TaSe}_{2}$ (2-D) and of rhodium (3-D) metal. In these metals, the Fermi discontinuity is clearly observed and, as the energy resolution is better than $20 \mathrm{meV}$, the width at $\mathrm{E}_{\mathrm{F}}$ is essentially due to the thermal broadening.

\subsection{Discussion of the possible mechanisms}

In order to look for an explaination of the striking absence of a Fermi step in the photoemission spectrum, we review the influence of the 1-D specificities (fluctuations, correlations) on the spectral function.

Let us first consider the influence of fluctuations on the spectral density. Mean field theory shows that, if fluctuations are ignored, a second-order transition takes place at $\mathrm{T}_{\mathrm{P}} \mathrm{MF}$ (mean field Peierls temperature). In strictly 1-D materials, fluctuations suppress the transition and the energy gap fluctuates in time and space below $T_{P}{ }^{M F}$. This behaviour leads to a pseudo-gap in the density of excitations near $E_{F}$ and then to a strong reduction of the spectral intensity at $E_{F}$ [5]. To account for a transition at a finite temperature in real materials, the transverse coupling must be considered. It yields a reduction of the fluctuations and the system undergoes a transition at $T_{P}\left(0<T_{P} « T_{P}{ }^{M F}\right)$. Rice et al. have investigated the combined influence of fluctuations and transverse coupling on the density of excitations [11]. Their analysis shows that the fluctuations induce the formation above $T_{P}$ of a pseudo-gap reminiscent of the actual gap in the low-temperature insulating phase. Such a pseudo-gap was observed at room temperature in $\mathrm{K}_{0.3} \mathrm{MoO}_{3}$ and $\left(\mathrm{TaSe}_{4}\right)_{2} \mathrm{I}$ by optical measurements $[8,12]$. Moreover this pseudo-gap is accompanied by a peak of quasi-particle states centered at $\Delta$ (half-gap). As such a peak is not actually observed (figure 3 ) and as measurements up to room temperature do not restore the metallic signature, one has to look for additional mechanisms contributing to the spectral weight reduction near $\mathrm{E}_{\mathrm{F}}$.

As mentioned in the introduction, correlations in 1-D are highly singular. In contrast to metals in higher dimension, correlations cannot be treated in perturbation and the excitation spectrum is completely different: while Fermi liquids are dominated at lowenergy by quasi-particle type excitations, only collective excitations are present in the 1-D correlated electron systems (Luttinger liquid). In strongly correlated Fermi liquids, the spectral density significantly differs from the density of quasi-particle states. The spectral function $\rho\left(E_{F}\right)$ and the quasi-particle density of states $N\left(E_{F}\right)$ at the Fermi level are related by the renormalization factor $Z$ through the relation $\rho\left(E_{F}\right)=Z N\left(E_{F}\right)$. Therefore, the lower the renormalization factor, the lower the spectral function at $E_{F}$. This factor $Z$ is also the discontinuity amplitude in the momentum distribution function at Fermi momentum in such systems. In 1-D metals the discontinuity disappears [4] and the singular limit $\mathrm{Z} \rightarrow>0$ must be considered. In this limit, the representation of excitations in terms of quasiparticles is no longer valid: the state of electrons on the Fermi surface becomes unstable with respect to the emission of electron-hole pairs. A classical example of such an infrared 
catastrophe is encountered in the edge singularity of $X$-ray absorption spectroscopy and results in a vanishing spectral weight at the edge [13]. Then, we expect the infrared catastrophe in 1-D system to induce a strong reduction of intensity near $E_{F}$. This conjecture is corroborated by a calculation in the Luttinger model which predicts a vanishing spectral density at $E_{F}[14]$.

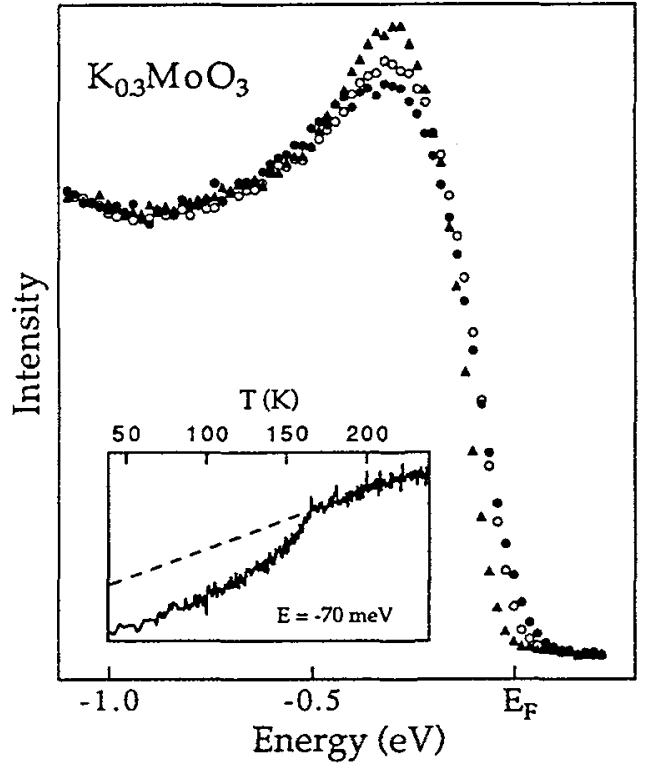

Figure 3: UPS spectrum at several temperatures. inset: $T$-dependence of the intensity at $E=-70 \mathrm{meV}$.

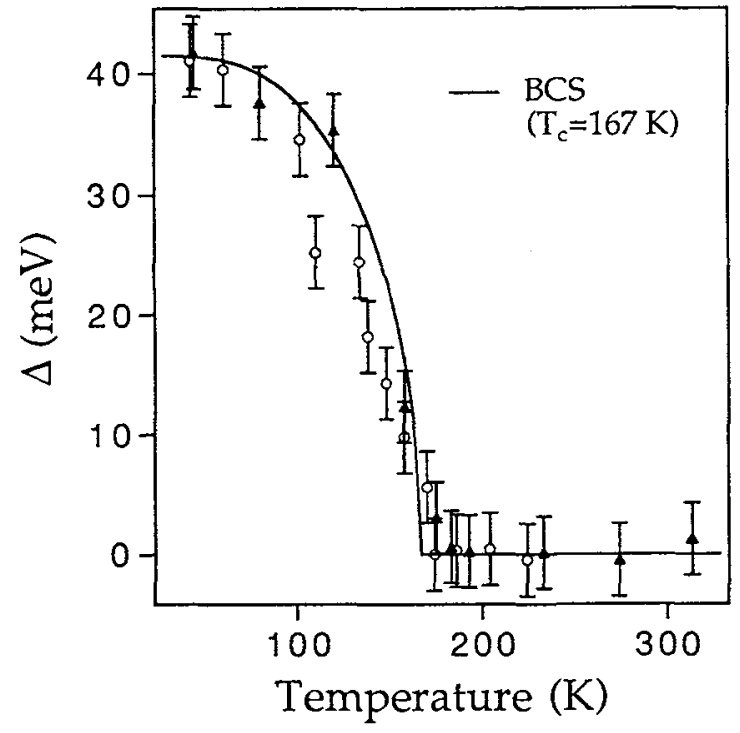

Figure 4: T-dependence of $\Delta$. The solid line is the gap in BCS model.

\subsection{The Peierls transition in the blue bronze}

In figure 3, we have reported the $\mathrm{He} I$ photoemission spectra of $\mathrm{K}_{0.3} \mathrm{MoO}_{3}$ for different temperatures. These spectra, normalized to their area, are all characterized by a pronounced structure centered at about $-300 \mathrm{meV}$ and a low spectral density near $\mathrm{E}_{\mathrm{F}}$. With decreasing temperature, a deformation of the spectrum is shown, with an additional shift for temperatures below the transition temperature ( $T_{P}=180 \mathrm{~K}$ ) [15]. This behaviour can be interpreted by a transfer of spectral weight from the vicinity of the Fermi level to higher binding energies. Moreover, the spectral function is modified over a much wider energy range $(500 \mathrm{meV}$ ) than expected from the opening of a Peierls gap (50-75 eV). In the inset, the photoemission intensity at constant binding energy $(E=-70 \mathrm{meV})$ is reported as a function of temperature. The spectral intensity slowly decreases with decreasing temperature in the high temperature range, and exhibits a sudden change of slope at $T=167 \mathrm{~K}$ which is unambiguously correlated with the electronic transition of this compound. The characteristic temperature $(167 \mathrm{~K})$ is slightly reduced with respect to the nominal transition temperature $\left(T_{c}=180 \mathrm{~K}\right)$. Surface effects could be responsible for this observation. In order to investigate the modification of the electronic structure below $T_{c}$, we define a phenomenological parameter $\Delta(T)$, which is the shift of the inflection point of the 
photoemission spectrum with respect to its position at $\mathrm{T}=183 \mathrm{~K}(\mathrm{E}=-80 \mathrm{meV})$. Above $167 \mathrm{~K}$, $\Delta$ does not significantly vary with temperature whereas below $167 \mathrm{~K}$ a rapid evolution is observed as shown in figure 4. Moreover, the solid line shows that $\Delta(T)$ roughly follows the temperature dependence of the gap in a BCS model. This dependence suggests that this parameter is in some way correlated with the Peierls gap but cannot be identified with it since the evolution of the spectra through the phase transition does not evidence the lowenergy feature corresponding to the Peierls gap. Then we can conclude that the photoemission spectra indirectly reveal the Peierls gap opening in this CDW system.

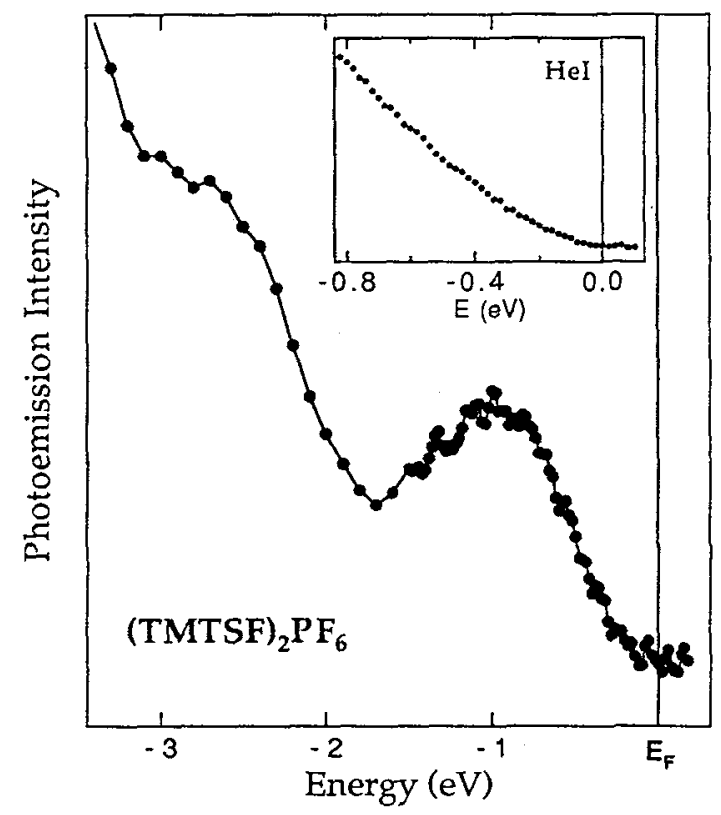

Figure 5: Photoemission spectrum of (TMTSF) ${ }_{2} \mathrm{PF}_{6}$ at $\mathrm{T}=50 \mathrm{~K}$. In the insert, the spectrum near $E_{F}$ clearly shows, with a better statistics, that no Fermi edge is detectable and that the correlation exponent is larger than 1.

\section{Organic $1 \mathrm{D}$ compounds}

In inorganic systems, the relative importance of the different mechanisms proposed in section 2.2 cannot be discriminated and it is possible that the physical properties result from a complex interplay of them. It is therefore important to examine materials where one of these mechanisms dominates. We have performed a photoemission study of a series of $1-\mathrm{D}$ organic compounds: (TMTSF) $2 \mathrm{X}$ with $\mathrm{X}=\mathrm{ClO}_{4}, \mathrm{PF}_{6}$ etc. These materials generally behave as good metallic conductors and exhibit at low temperature (below 12K) a spin-density-waves phase suggesting the large strength of electronic correlations [16,17]. Thus, in contrast to the CDW inorganic materials where electron-phonon interactions are important, electronic correlations play the dominant role. Moreover, critical fluctuations associated with the low-temperature transition are expected to be small in the metallic temperature range. Figure 5 shows the photoemission spectrum of $\left(\mathrm{TMTSF}_{2} \mathrm{PF}_{6}\right.$ at $50 \mathrm{~K}$ in its metallic phase. It is dominated by broad structures with the shallowest one at $-1.2 \mathrm{eV}$ and a very weak spectral weight is found at $E_{F}$. A close up of the region near $E_{F}$ clearly shows that no Fermi edge is detectable, as previously observed in 1D inorganic compounds. Tomonaga and Luttinger [4] have proposed a simple, exactly solvable, model for interacting 
1D electrons with a linearised dispersion. The salient features of the model are: a) the absence of quasi-particules, b) spin-charge separation and c) decay, with non-universal power laws, of all correlation functions. As an example, the integrated spectral function vanishes at the Fermi energy according to the asymptotic power law $\rho(\omega) \sim \omega^{\alpha}$, where $\alpha$ reflects the coupling strength. The comparison of this power law with experimental data suggests that $\alpha>1$. This very large value of $\alpha$ is consistent with recent NMR measurements [18] but reveal the presence of large electronic correlations which could be difficult to conciliate with a metallic state [19]. Additional studies are required to clarify this point.

\section{Conclusion}

In this study, we have used high-resolution photoemission for the study of low excitations in 1-D materials. In metallic phases we observed the absence of a Fermi edge, a behaviour seems to result from the singularities of one-dimension. Moreover, the modification of the electronic structure accompanying the $\mathrm{CDW}$ transition in $\mathrm{K}_{0.3} \mathrm{MoO}_{3}$ are directly reflected in the photoemission spectra near the Fermi level. Such measurements show that owing to the high-resolution, the low-energy excitations which govern the physical properties of solids, are now accessible to the field of electron spectroscopies.

\section{References}

1. R. F. Peierls, Quantum theory of solids (Clarendon Press, Oxford 1955).

3. L.D. Landau and E.M. Lifchitz, Statistical Physics, (Pergamon Press, 1959).

3. J. Solyom, Adv. Phys. 28 (1979) 201.

4. J.M. Luttinger, J. Math. Phys. 4 (1963) 1154.

5. P.A. Lee, M. Rice and P.W. Anderson, Phys. Rev. Lett. 31 (1973) 462.

6. Low dimensional electronic properties of molybdenum bronzes and oxides, edited by C. Schlenker (Kluwer Academic Publishers, Dordrecht, 1989).

7. J.P. Pouget, C. Schlenker and J. Marcus, J. Phys. Lett. 44 (1984) L113.

8. G. Travaglini, P. Wachter, J. Marcus and C. Schlenker, Solid State Comm. 37 (1981) 599.

9. M.H. Wangboo and L.F. Schneemeyer, Inorg. Chem. 25 (1986) 2424.

10. B. Dardel, D. Malterre, M. Grioni, P. Weibel, Y. Baer and F. Lévy, Phys. Rev. Lett. 67 (1991) 3144.

11. M.J. Rice and S. Strässler, Solid State Commun. 13 (1973) 1389.

12. H.P. Geserich, G. Scheiber, M. Dürrler, F. Lévy and P. Monceau, Physica 143B (1986) 198.

13. P.W. Anderson, Phys. Rev. Lett. 18 (1967) 1049.

14. H.J. Schulz, Int. J. Mod. Phys. 5 (1991) 57.

15. B. Dardel et al, Europhys. Left. 19, 525 (1992).

16. D. Jérome and H.J. Schulz, Adv. Phys. 31 (1982) 299.

17. K. Bechgaard and D. Jérome, Phys. Scripta T39 (1991) 37.

18. P. Wzietek et al., J. Physique I (France) 3 (1993) 171.

19. F. Mila and $X$.Zotos, to be published 